\title{
Bone marrow-derived circulating progenitor cells fail to transdifferentiate into adipocytes in adult adipose tissues in mice
}

\author{
Young Jun Koh, ${ }^{1}$ Shinae Kang, ${ }^{1}$ Hyuek Jong Lee, ${ }^{1}$ Tae-Saeng Choi, ${ }^{2}$ \\ Ho Sub Lee, ${ }^{3}$ Chung-Hyun Cho, ${ }^{4}$ and Gou Young Koh ${ }^{1}$ \\ ${ }^{1}$ National Research Laboratory of Vascular Biology and Department of Biological Sciences, \\ Korea Advanced Institute of Science and Technology, Daejeon, Republic of Korea. ${ }^{2}$ Department of Microbiology, \\ College of Medicine, Dankook University, Chonan, Republic of Korea. ${ }^{3}$ Department of Physiology, College of Oriental Medicine, \\ Wonkwang University, Iksan, Republic of Korea. ${ }^{4}$ Department of Physiology, College of Medicine, Choongnam University, Daejeon, Republic of Korea.
}

\begin{abstract}
Little is known about whether bone marrow-derived circulating progenitor cells (BMDCPCs) can transdifferentiate into adipocytes in adipose tissues or play a role in expanding adipocyte number during adipose tissue growth. Using a mouse bone marrow transplantation model, we addressed whether BMDCPCs can transdifferentiate into adipocytes under standard conditions as well as in the settings of diet-induced obesity, rosiglitazone treatment, and exposure to G-CSF. We also addressed the possibility of transdifferentiation to adipocytes in a murine parabiosis model. In each of these settings, our findings indicated that BMDCPCs did not transdifferentiate into either unilocular or multilocular adipocytes in adipose tissues. Most BMDCPCs became resident and phagocytic macrophages in adipose tissues - which resembled transdifferentiated multilocular adipocytes by appearance, but displayed cell surface markers characteristic for macrophages - in the absence of adipocyte marker expression. When exposed to adipogenic medium in vitro, bone marrow cells differentiated into multilocular, but not unilocular, adipocytes, but transdifferentiation was not observed in vivo, even in the contexts of adipose tissue regrowth or dermal wound healing. Our results suggest that BMDCPCs do not transdifferentiate into adipocytes in vivo and play little, if any, role in expanding the number of adipocytes during the growth of adipose tissues.
\end{abstract}

\section{Introduction}

Balanced growth of adipose tissues is necessary for energy homeostasis, insulation of heat loss, and mechanical support for other structures in the body. However, excess adipose tissue is defined as obesity and is associated with metabolic syndrome $(1,2)$. Growth of adipose tissues mainly results from an increase in the number and size of adipocytes $(3,4)$. During embryonic development, some mesenchymal stem cells differentiate into adipoblasts that eventually become preadipocytes. These preadipocytes become unilocular, lipid-laden mature adipocytes by appropriate stimulation during postnatal development. This sequential differentiation process from mesenchymal stem cells to mature adipocytes is termed adipogenesis (5). In comparison, under a higher level of energy homeostasis, mature adipocytes become bigger adipocytes with an unilocular accumulation of lipid, a process known as lipogenesis (6). Adipogenesis is the predominant process in the normal development of adipose tissues after birth, while lipogenesis is the predominant process in the development of obesity involving adult adipose tissues. These 2 fundamental processes have been thought to mainly occur in the resident adipoblasts, preadipocytes, and adipocytes within each area of adipose tissue, although there is a lack of clear evidence that this is so.

Nonstandard abbreviations used: BMDCPC, bone marrow-derived circulating progenitor cell; BMT, bone marrow transplantation; DPBS, Dulbecco's PBS; LYVE-1, lymph vessel endothelial hyaluronan receptor-1; ROSI, rosiglitazone; SVC, stromal vascular cell.

Conflict of interest: The authors have declared that no conflict of interest exists. Citation for this article: J. Clin. Invest. 117:3684-3695 (2007). doi:10.1172/JCI32504
Adult white adipose tissue is mainly composed of mature unilocular (i.e., comprising a single lipid compartment surrounded by perilipin) adipocytes, which are supported by stromal vascular tissue containing vascular endothelial cells and macrophages, and poorly characterized undifferentiated or stem cells containing adipoblasts, preadipocytes, fibroblasts, and hematopoietic cells (7-11). Obviously, the sizes, shapes, numbers, and even roles of these cells are variable depending on their localization and situation during overgrowth of adipose tissues, i.e., obesity $(12,13)$. In obesity, a size increase in adipocytes is a common and evident phenomenon. Rodents fed a high-fat diet from adulthood display abnormal overgrowth of adipose tissues with variable increases in adipocyte size, a consequence of lipogenesis. This manifestation is followed by variable increases in the number of adipocytes, a consequence of proliferation of resident adipoblasts/preadipocytes (3, $4,14)$. However, it is still not known whether these resident adipoblasts and preadipocytes are derived from mesenchymal cells within adipose tissue (9) or from bone marrow stem cells outside of adipose tissue through circulation and recruitment as bone marrow-derived circulating progenitor cells (BMDCPCs). Moreover, because there are still no specific molecular markers to distinguish adipoblasts and preadipocytes from differentiated adipocytes and nonadipocytes in the adult adipose tissue, the underlying mechanisms of how the number of adipocytes increases during adipose tissue growth remains to be elucidated.

BMDCPCs originate from hematopoietic stem cells and mesenchymal stem cells in the bone marrow and are mobilized from bone marrow to systemic circulation under appropriate stimuli. These BMDCPCs become resident cells in every organ, 


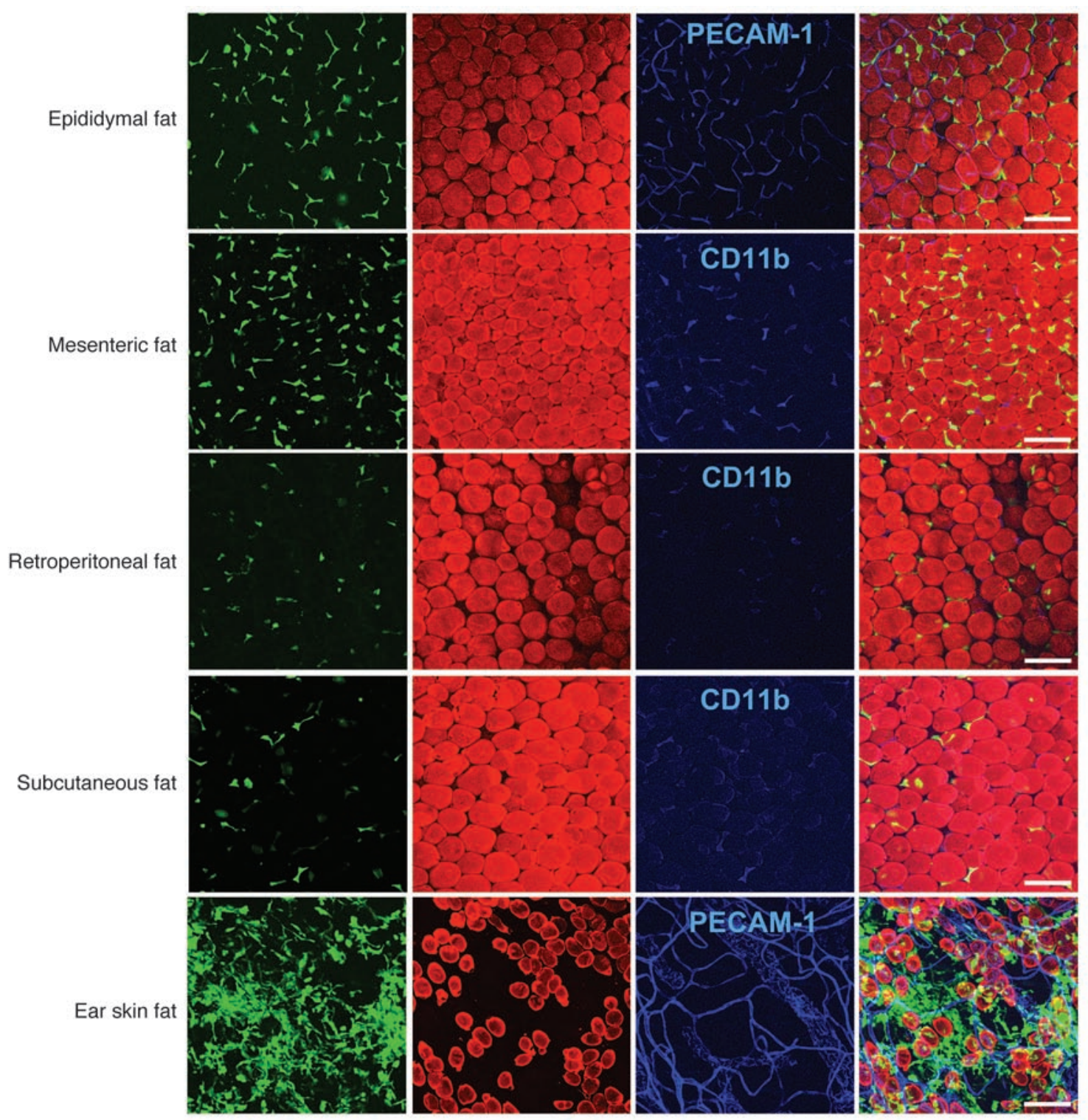

\section{Figure 1}

No GFP+perilipin ${ }^{+}$adipocytes were detected in the adipose tissues of mice fed a normal diet. Adipose tissues were harvested from C57BL/6J mice that had received BMT from GFP+ mice 2 months previously and then fed a normal diet for the 2-month period. Tissues were whole-mounted, coimmunostained for perilipin (adipocytes; red stain) and PECAM-1 or CD11b (blood vessels or macrophages, respectively; blue stain), and merged. No GFP ${ }^{+}$cells (green stain) were perilipin ${ }^{+}$adipocytes in the adipose tissues; approximately half of the GFP+ cells were CD11 $\mathrm{b}^{+}$ macrophages in the adipose tissues. Scale bars: $100 \mu \mathrm{m}$.

variously as differentiated or transdifferentiated cells depending on the local tissue microenvironment (15-23). However, whether BMDCPCs can transdifferentiate into mature adipocytes in adult adipose tissues has not been well established. Moreover, it is unknown whether BMDCPCs play a significant role in expanding the number of adipocytes in the growth of adipose tissues. Therefore, in this study, we investigated whether BMDCPCs can transdifferentiate into adipocytes in normal and obese adipose tissues. To do so, we used visualization and characterization of $\mathrm{GFP}^{+}$cells in the several adipose tissues of the adult mice that had received bone marrow transplantation (BMT) with donor bone marrow cells from transgenic mice expressing enhanced GFP on a universal promoter (24). We also questioned whether BMDCPCs can transdifferentiate into adipocytes in the regrowing adipose tissue and in wound-healing dermal tissue. To clearly visualize and characterize $\mathrm{GFP}^{+} \mathrm{BMDCPC}$ in the multicellular adipose tissues, whole-mounted adipose tissues were immunostained for markers of adipocytes, macrophages, and extracellular matrix protein.

Our results with rigorous confocal microscopic analysis revealed that BMDCPCs did not transdifferentiate into unilocular or multilocular adipocytes in normal, obese, or regrowing adipose tissues. During preparation of this article, Crossno et al. (25) reported that BMDCPCs can transdifferentiate into multilocular, but not unilocular, adipocytes in the adipose tissues. Given these different 

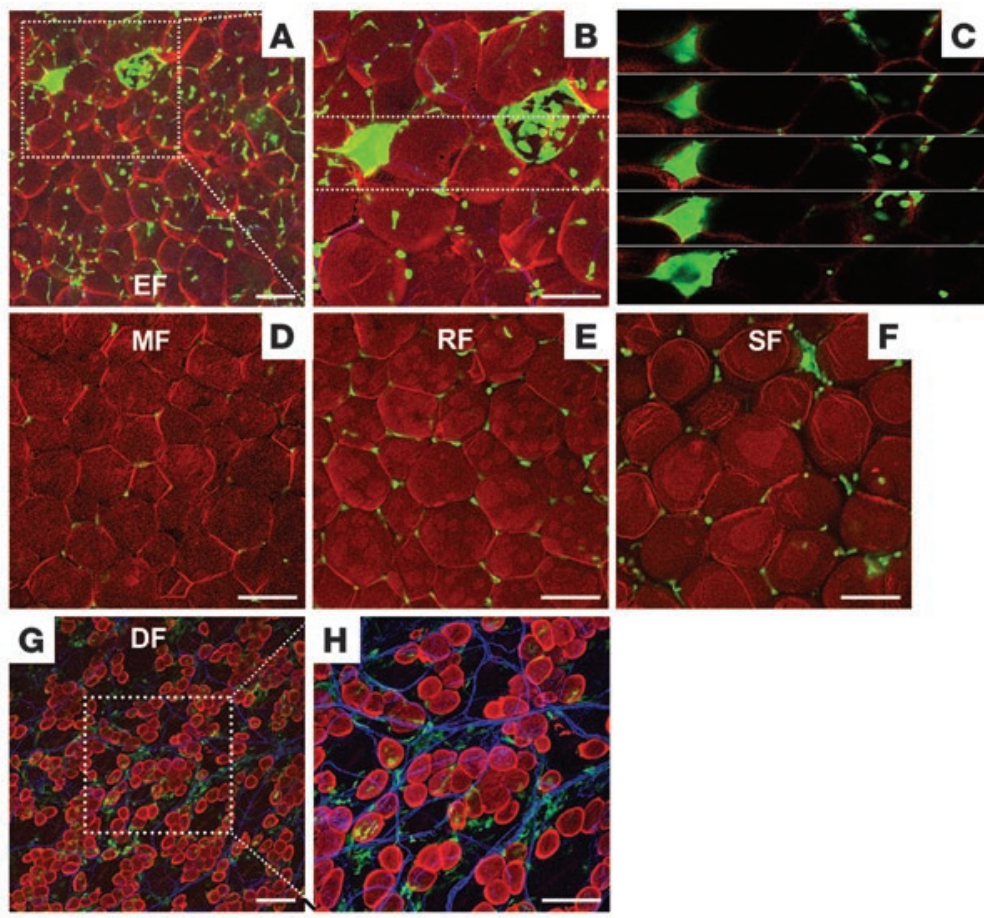

\section{Figure 2}

No GFP+perilipin ${ }^{+}$adipocytes were detected in the adipose tissues of mice fed a high-fat diet. Adipose tissues were harvested from C57BL/6J mice that had received BMT from GFP+ mice 2 months previously and then were fed a high-fat diet for the 2-month period. Tissues were whole-mounted, coimmunostained for perilipin (red stain) and PECAM-1 (blue stain), and merged. The sizes of adipocytes were approximately twice that of adipocytes from the mice fed the normal diet. (A-C) In epididymal fat (EF), some larger GFP+ cells (green stain) looked like unilocular adipocytes ( $\mathbf{A}$ and $\mathbf{B}$, boxed regions), but sequential dissected images of this portion (boxed region in B) revealed clustered GFP+perilipin- smaller cells (C). (D) Mesenteric fat (MF). (E) Retroperitoneal fat (RF). (F) Subcutaneous fat (SF). ( $\mathbf{G}$ and $\mathbf{H}$ ) Dermal fat of ear skin (DF); $\mathbf{H}$ shows a higher-magnification view of boxed region in G. No GFP+ cells were perilipin+ adipocytes in the adipose tissues. Scale bars: $100 \mu \mathrm{m}$. findings, we discuss our results in the context of the similarities and dissimilarities between these 2 studies.

\section{Results}

BMDCPCs do not transdifferentiate into adipocytes in adult adipose tissues under normal or obese conditions. To investigate the possibility of a commitment of BMDCPCs to transdifferentiate into adipocytes in adult adipose tissues, we examined the adipose tissues of BMT mice transplanted 2 months previously with $\mathrm{GFP}^{+}$bone marrow cells. To detect $\mathrm{GFP}^{+}$adipocytes in the adipose tissues, the wholemounted adipose tissues were coimmunostained for perilipin (a membrane protein that surrounds lipid droplets and is selectively expressed in adipocytes and steroidogenic cells; refs. 26, 27), adipophilin (known as adipose differentiation-related protein; a membrane protein that surrounds small lipid droplets and is expressed ubiquitously; refs. 26-28) and CD11b (a specific marker of monocytes/macrophages) or PECAM-1 (a specific component of vascular endothelial cells). Our analysis revealed that none of the $\mathrm{GFP}^{+}$cells were perilipin ${ }^{+}$adipocytes in epididymal ( $\sim 50,000$ adipocytes), mesenteric ( $\sim 30,000$ adipocytes), retroperitoneal $(\sim 20,000$ adipocytes), subcutaneous ( $\sim 50,000$ adipocytes), or dermal $(\sim 5,000$ adipocytes) adipose tissues of the normal diet-fed mice $(n=5$; Figure 1 and Supplemental Figure 1A; supplemental material available online with this article; doi:10.1172/JCI32504DS1). Moreover, none of the $\mathrm{GFP}^{+}$cells were adipophilin ${ }^{+}$adipocytes in epididymal ( 2,500 adipocytes) or retroperitoneal ( $\sim 1,000$ adipocytes) adipose tissues of the normal diet-fed mice $(n=3$; Supplemental Figures $1 \mathrm{~B}$ and $2 \mathrm{~A}$ ). Most $\mathrm{GFP}^{+}$cells were $\mathrm{CD} 11 \mathrm{~b}^{+}, \mathrm{F} 4 / 80^{+}$, lymph vessel endothelial hyaluronan receptor-1-positive (LYVE-1 $1^{+}$), or $\mathrm{CD}_{45^{+}}$macrophages in the adipose tissues (Figure 1 and Supplemental Figure 3). In comparison, bone marrow-derived GFP ${ }^{+}$ cells were largely detected as macrophages or leukocytes in several organs including spleen, kidney, lung, intestinal villi, liver, tracheal mucosa, and retina (Supplemental Figure 4). Sometimes, groups of $\mathrm{GFP}^{+}$cells were detected, but a dissecting image analysis of these cells by confocal microscopy revealed that these cells clearly were not $\mathrm{GFP}^{+}$adipocytes (Supplemental Figure 5).

To examine the commitment, if any, of BMDCPCs to transdifferentiate into adipocytes of adult adipose tissues during obesity, the BMT mice that had received GFP $\mathrm{GF}^{+}$bone marrow cells 2 months previously were fed with a high-fat diet containing $34.9 \%$ (wt/wt) fat for the 2 months following BMT. The high-fat diet-fed mice displayed an approximately 1.4 -fold increase in body weight, an approximately 2.2 -fold increase in epididymal adipose tissue weight, and an approximately 2.0 -fold increase in the diameter of adipocytes in epididymal adipose tissue compared with mice fed normal diet (Figure 2 and Supplemental Figure 6). Nevertheless, our analysis revealed that none of the $\mathrm{GFP}^{+}$cells were perilipin ${ }^{+}$adipocytes in epididymal ( $\sim 50,000$ adipocytes $)$, mesenteric $(\sim 30,000$ adipocytes), retroperitoneal ( $\sim 20,000$ adipocytes), subcutaneous $(\sim 50,000$ adipocytes), or dermal ( $\sim 5,000$ adipocytes) adipose tissues of the high-fat diet-fed mice $(n=5$; Figure 2 and Supplemental Figure $1 \mathrm{C})$. Moreover, none of the $\mathrm{GFP}^{+}$cells were adipophilin ${ }^{+}$adipocytes in epididymal ( 2,500 adipocytes) or retroperitoneal $(\sim 1,000$ adipocytes) adipose tissues of the high-fat diet-fed mice $(n=3$; Supplemental Figure 2B). Most of the $\mathrm{GFP}^{+}$cells were small, singular nonadipocytes in the adipose tissues (Figure 2$)$. Several ( 750 of 155,000 adipocytes) $\mathrm{GFP}^{+}$cells that were similar to unilocular adipocytes were detected (Figure 2, A and B); however, a dissecting image analysis of these cells by confocal microscopy revealed that these cells were not $\mathrm{GFP}^{+}$unilocular adipocytes but instead were clustered $\mathrm{GFP}^{+}$cells (Figure 2C). In fact, most of these cells were $\mathrm{F} 4 / 80^{+}$macrophages ( $85 \%$; Figure $\left.3, \mathrm{~A}-\mathrm{C}\right)$, and to a lesser extent, CD $11 \mathrm{~b}^{+}$macrophages $(26 \%)$ or $\mathrm{CD} 45^{+}$macrophages $(-34 \%$; Figure $3, \mathrm{~A}$ and $\mathrm{B})$. However, these cells contained a negligible number of LYVE- $1^{+}$macrophages ( $4 \%$; Figure 3 , A and B). Thus, our results indicate that BMDCPCs do not transdifferentiate into adipocytes in adult adipose tissues under normal or obese conditions. 
A
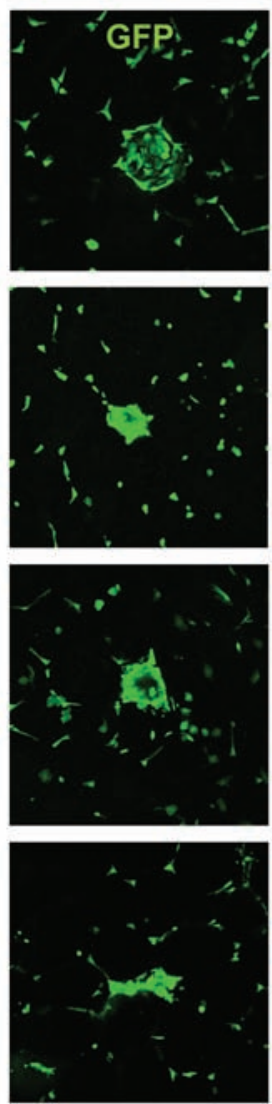
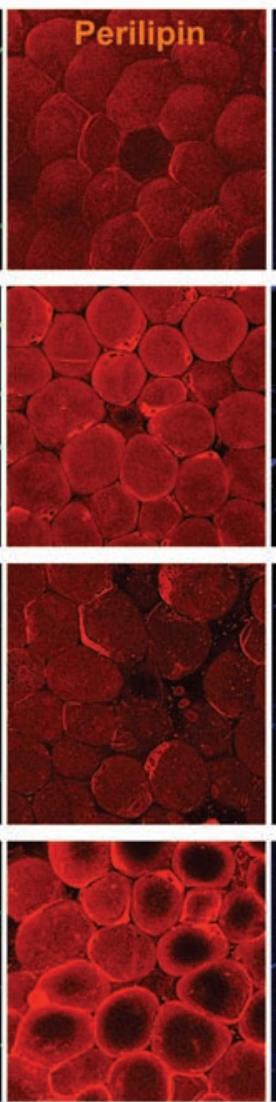
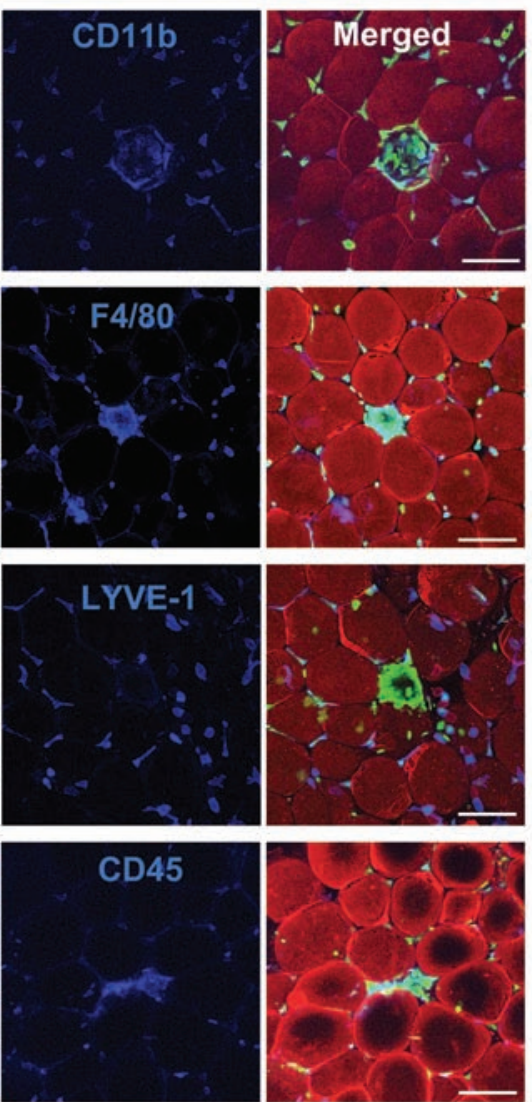
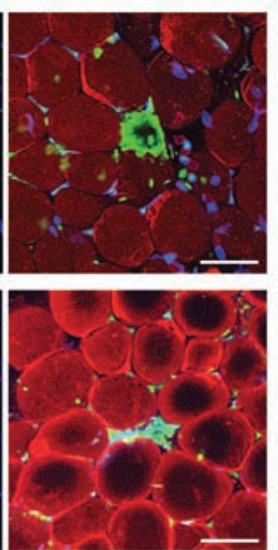

B
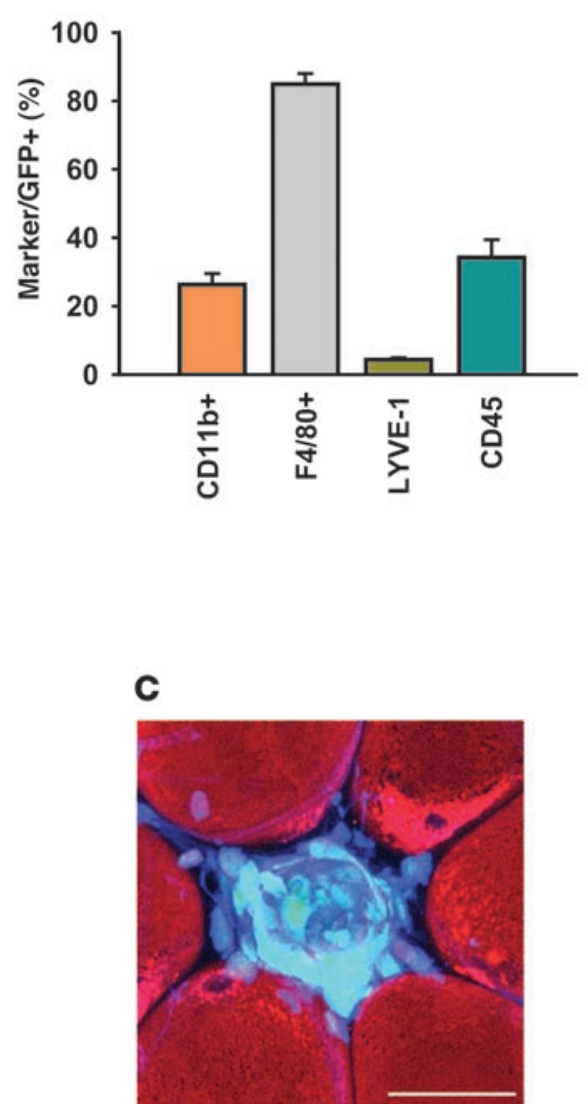

\section{Figure 3}

Clustered GFP+ cells in the adipose tissue of mice fed the high-fat diet were F4/80+ macrophages. (A) Epididymal adipose tissue was harvested from C57BL/6J mice that had received BMT from GFP+ mice 2 months previously and then were fed a high-fat diet for the 2-month period. Tissues were whole-mounted; coimmunostained for perilipin (red stain) and the macrophage markers CD11b, F4/80, LYVE-1, or CD45 (blue stain); and merged. Note that most of the clustered GFP+ cells (green stain) were F4/80+ macrophages, and to a lesser extent, these were CD11 $\mathrm{b}^{+}$or CD45+ macrophages. Scale bar: $100 \mu \mathrm{m}$. (B) GFP+CD11b+, GFP+F4/80+, GFP+LYVE-1+, and GFP+CD45+ macrophages were counted in 30 regions of clustered GFP+ cells $(\sim 3,000)$ and presented as a percentage of the total counted, clustered GFP ${ }^{+}$cells. (C) Higher-magnification view of the merged GFP-, perilipin-, and F4/80-stained (green, red, and blue stain, respectively) image in A revealed characteristic GFP+F4/80+ clustered macrophages. Scale bars: $50 \mu \mathrm{m}$.

BMDCPCs do not transdifferentiate into unilocular or multilocular adipocytes in adult adipose tissues in normal or obese conditions. Because a recent study (25) reported that high-fat diet-induced obesity promoted transdifferentiation of BMDCPCs into multilocular adipocytes in the adipose tissues, with a range of about $7 \%-17 \%$ of total adipocytes, we determined whether $\mathrm{GFP}^{+}$perilipin ${ }^{+}$multilocular adipocytes were formed in the normal and high-fat diet-induced obese adipose tissues. To distinguish multilocular adipocytes from unilocular adipocytes in adult adipose, the whole-mounted adipose tissues were coimmunostained for perilipin and collagen IV, which surrounds each adipocyte individually as a basement membrane (29). This coimmunostaining clearly distinguished not only collagen IV-covered adipocytes from nonadipocytes, but also multilocular adipocytes from unilocular adipocytes (Figure 4). Compared with the adipose tissues of normal diet-fed mice, the adipose tissues from mice consuming a high-fat diet for 2 months did not exhibit a significant change in the extent of multilocularization of adipocytes. In both normal and high-fat diet-induced obese mice, the population frequency of multilocu- lar adipocytes in white adipose tissues was as follows, arranged in descending order of frequency: mesenteric (25\%-27\%), retroperitoneal (20\%-23\%), dermal (11\%-14\%), subcutaneous ( $8 \%-10 \%)$, epididymal (3\%-4\%). In comparison, most of the adipocytes in the intersubscapular brown adipose tissues were multilocular adipocytes in both groups. Nevertheless, in contrast to the report of Crossno et al. (25), none of the $\mathrm{GFP}^{+}$cells were perilipin ${ }^{+}$multilocular adipocytes in epididymal ( $\sim 12,000$ adipocytes $)$, mesenteric ( $\sim 24,000$ adipocytes), retroperitoneal ( $\sim 24,000$ adipocytes), subcutaneous ( $\sim 18,000$ adipocytes), or dermal ( $\sim 3,000$ adipocytes) adipose tissues of the normal and high-fat diet-induced obese mice ( $n=3$ per group; Figure 4 ). In the obese mice, there were 2 kinds of clustered $\mathrm{GFP}^{+}$cells, $\mathrm{GFP}^{+}$perilipin ${ }^{-}$collagen $\mathrm{IV}^{+}$ and $\mathrm{GFP}^{+}$perilipin-collagen $\mathrm{IV}^{-}$(Figure $4 \mathrm{~B}$ ), which suggested that degradation of perilipin by phagocytic macrophages precedes degradation of collagen IV. In comparison, in the intersubscapular brown adipose tissue of the normal and obese mice, none of the $\mathrm{GFP}^{+}$cells were perilipin ${ }^{+}$multilocular adipocytes $(\sim 6,000$ adipocytes; $n=3$ per group; Figure 4$)$. It is interesting to 
A

GFP

Perilipin
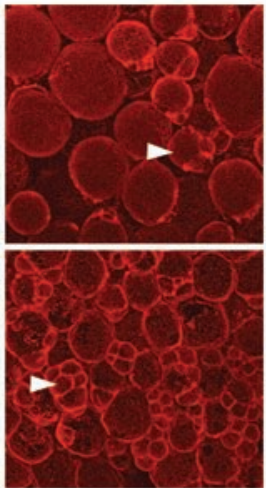

MF
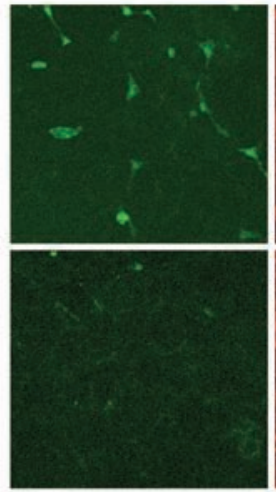

BF
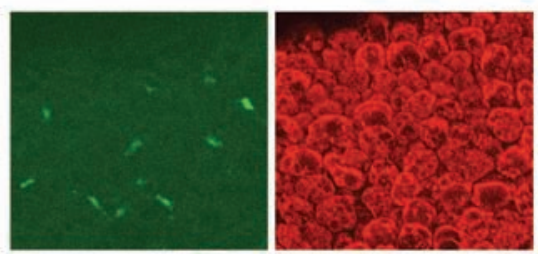

BF
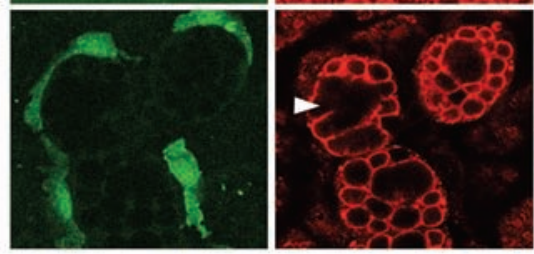

B
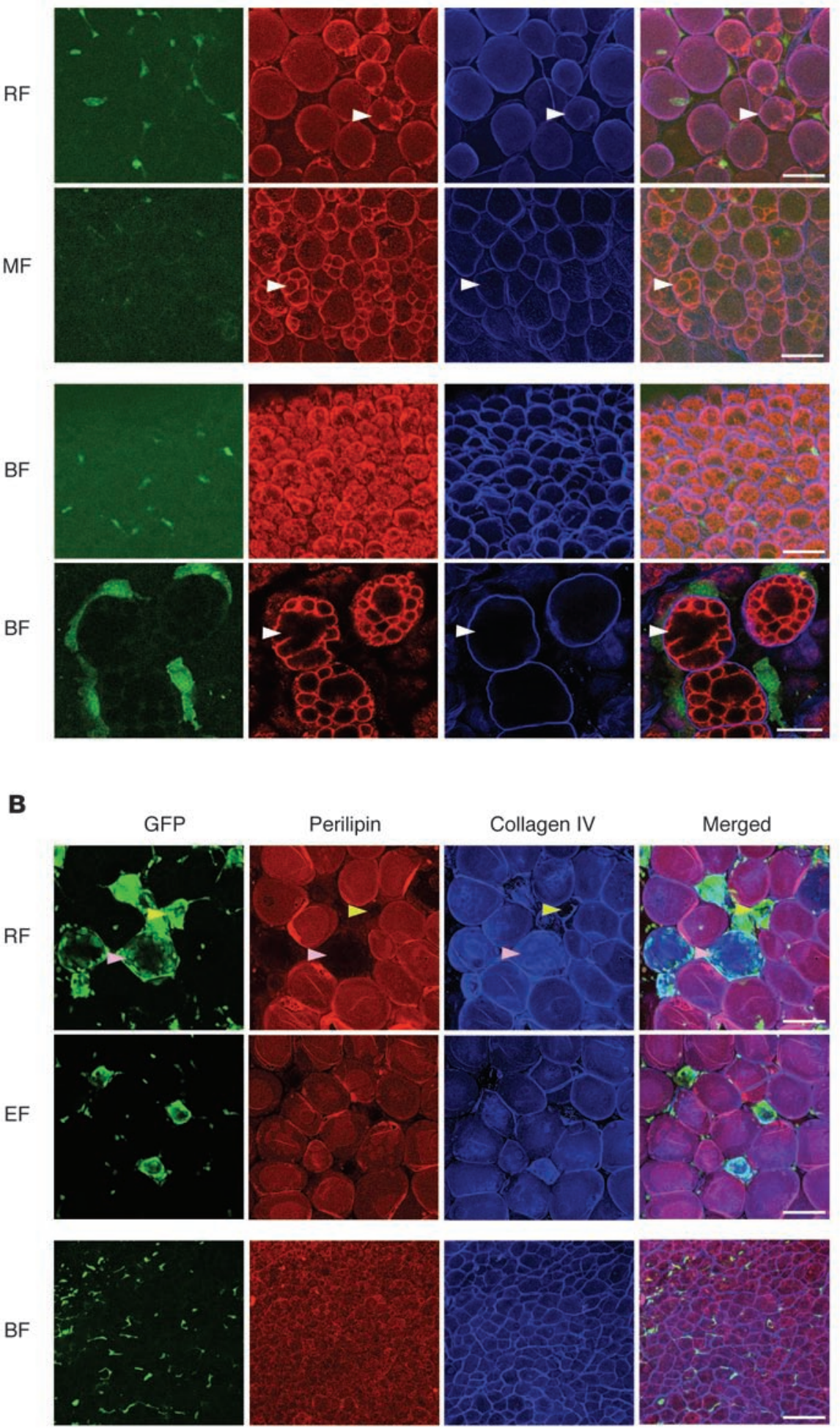
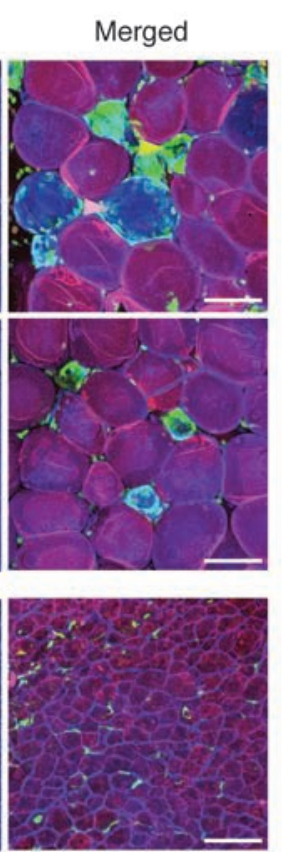

\section{Figure 4}

No GFP+perilipin+ unilocular and multilocular adipocytes were detected in the adipose tissues of mice fed normal or high-fat diet. Adipose tissues were harvested from C57BL/6J mice that had received BMT from GFP+ mice 2 months previously and were then fed a normal diet $(\mathbf{A})$ or a highfat diet (B) for the 2-month period. Tissues were whole-mounted, coimmunostained for perilipin and collagen IV (which surrounds each adipocyte individually as the basement membrane), and merged. White arrowheads in $\mathbf{A}$ indicate typical multilocular adipocytes. In $\mathbf{B}$, there are 2 kinds of clustered GFP+ cells: GFP+perilipin- collagen IV+ cells (pink arrowheads) and GFP+perilipin'-collagen IVcells (yellow arrowheads). Note that no GFP+ cells were perilipin+ multilocular or unilocular adipocytes. BF, intersubscapular brown fat. Scale bars: $50 \mu \mathrm{m}$ (A, top 3 rows); $20 \mu \mathrm{m}$ (A, bottom row); $100 \mu \mathrm{m}$ (B).

Rosiglitazone treatment does not induce transdifferentiation of BMDCPCs into multilocular adipocytes in adipose tissues. Crossno et al. (25) also reported that treatment with the pharmacological PPAR $\gamma$ activator rosiglitazone (ROSI) promoted transdifferentiation of BMDCPCs into multilocular adipocytes in the adipose tissues, ranging from $15 \%$ to $30 \%$ of total adipocytes. We performed the ROSI treatment with the same regimen (about $15 \mathrm{mg} / \mathrm{kg} / \mathrm{d}$ for 4 weeks) to examine whether $\mathrm{GFP}^{+}$perilipin ${ }^{+}$ multilocular adipocytes formed in the adipose tissue. However, in contrast to the report of Crossno et al. (25), none of the $\mathrm{GFP}^{+}$cells were perilipin ${ }^{+}$multilocular adipocytes in epididymal ( 40,000 adipocytes), mesenteric ( 24,000 adipocytes), retroperitoneal ( $\sim 16,000$ adipocytes), subcutaneous ( $\sim 40,000$ adipocytes), dermal $(\sim 4,000$ adipocytes), or intersubscapular brown ( $~ 8,000$ adipocytes) adipose tissues of the ROSI-treated mice $(n=4$; Figure 5 , $A$ and B). Intriguingly, the most unilocular adipocytes were instead in the epididymal, mesenteric, retroperitoneal, and subcutaneous tissues, but not in the dermal tissue; adipose tissues converted to the multilocular adipocytes (Figure 5, A and B). Similar to the Crossno et al. report (25), we sometimes observed numbers of massively clustered $\mathrm{GFP}^{+}$cells, but dissecting image analysis of these cells by confocal note that the clustered $\mathrm{GFP}^{+}$cells were rarely found in the intersubscapular brown adipose tissue, even in the obese mice. Thus, these results indicate that BMDCPCs do not transdifferentiate into either unilocular and multilocular adipocytes in adult adipose tissues under normal or obese conditions. microscopy revealed that they were not $\mathrm{GFP}^{+}$perilipin ${ }^{+}$multilocular adipocytes (Figure 5, C and D). Thus, the ROSI treatment did not induce transdifferentiation of BMDCPCs into multilocular adipocytes, although it massively induced a distinctive adipocyte multilocularization in several adipose tissues. 
A
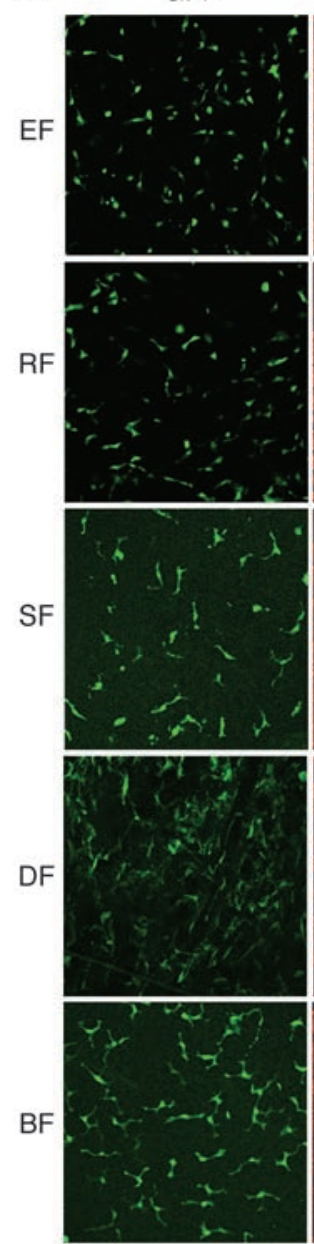

Perilipin
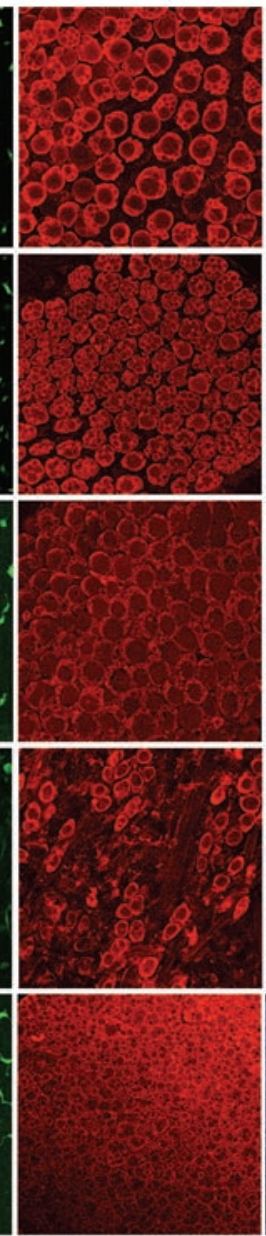

Collagen IV
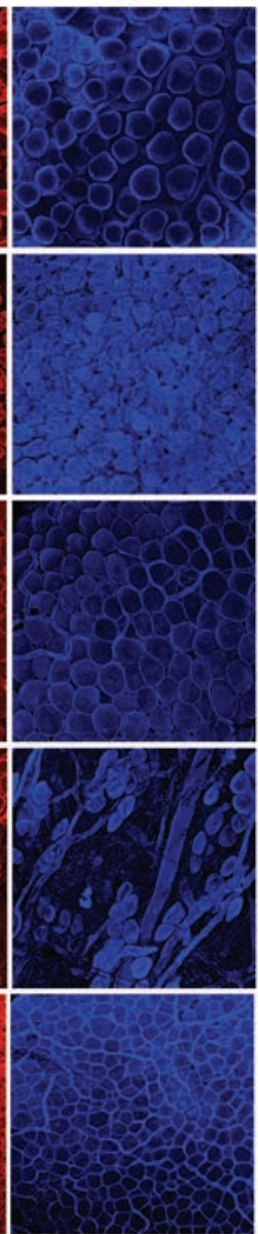

Merged
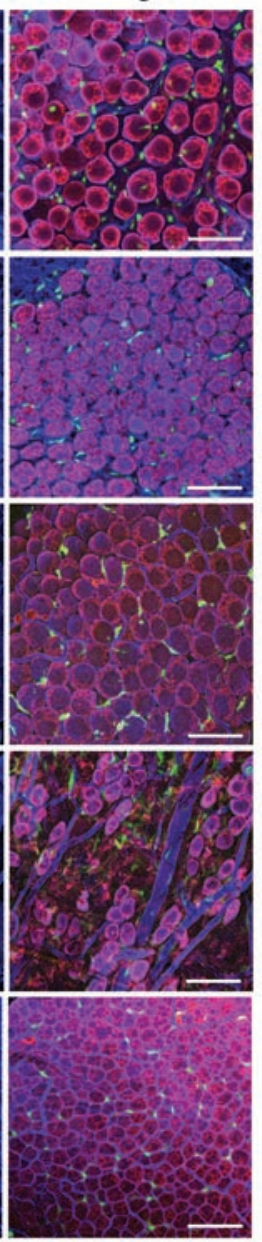

B
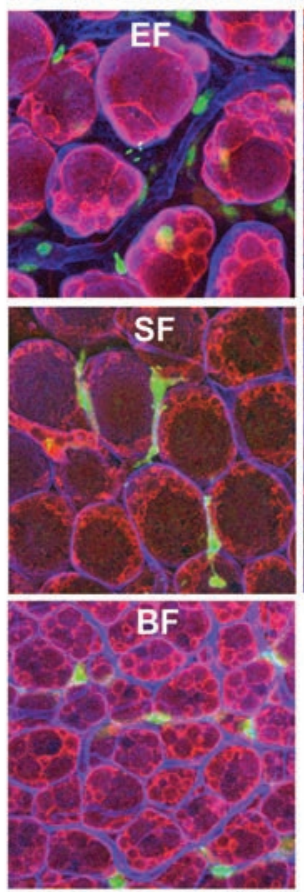

C

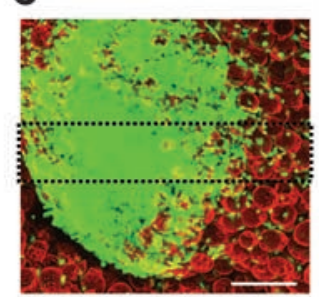

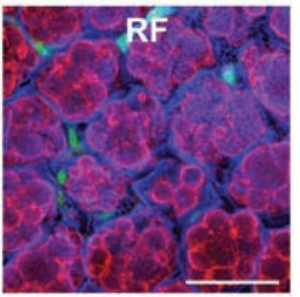

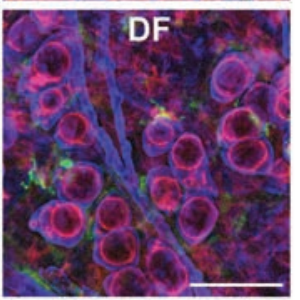

D

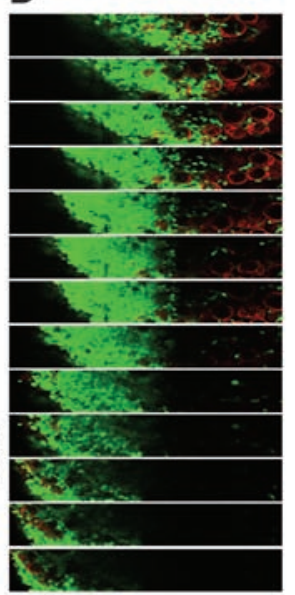

Figure 5

No GFP+perilipin+ unilocular and multilocular adipocytes were detected in the adipose tissues of mice treated with ROSI. Adipose tissues were harvested from C57BL/6J mice that had received BMT from GFP+ mice 2 months previously and then were treated with ROSI $(\sim 15 \mathrm{mg} / \mathrm{kg} / \mathrm{d})$ for 4 weeks. Tissues were whole-mounted, coimmunostained for perilipin and collagen IV (which surrounds each adipocyte individually as the basement membrane), and merged. (A and B) No GFP+perilipin+ unilocular and multilocular adipocytes were detected in the adipose tissues. Higher-magnifications views of the merged images in A revealed characteristic GFP-perilipin ${ }^{+}$multilocular adipocytes (B). (C and D) Massively clustered GFP+ cells were seen in epididymal fat, but sequential dissected images of the boxed region in $\mathbf{C}$ revealed that no GFP+ cells were perilipin $^{+}$adipocytes (D). Scale bars: $100 \mu \mathrm{m}$ (A and C); $50 \mu \mathrm{m}$ (B).

G-CSF treatment does not induce transdifferentiation of BMDCPCs into multilocular adipocytes in adipose tissues. Many previous studies (30-32) have demonstrated that adipocytes could be generated from bone marrow-derived mesenchymal stem cells in vitro, and G-CSF is known to mobilize both hematopoietic stem cells and mesenchymal stem cells $(33,34)$ from the bone marrow. For these reasons, we assessed any transdifferentiation of BMDCPCs into $\mathrm{GFP}^{+}$perilipin ${ }^{+}$ adipocytes in the adipose tissues of the mice, which were treated with G-CSF $(10 \mu \mathrm{g} / \mathrm{kg} / \mathrm{d}$ intraperitoneally for 2 weeks). However, none of the $\mathrm{GFP}^{+}$cells were perilipin ${ }^{+}$multilocular adipocytes in epididymal ( $\sim 30,000$ adipocytes), mesenteric ( 18,000 adipocytes), retroperitoneal ( $\sim 10,000$ adipocytes), subcutaneous $(\sim 30,000$ adipocytes), or intersubscapular brown ( $\sim 6,000$ adipocytes) adipose tissues of the G-CSF-treated mice $(n=3)$ (Supplemental Figure 7). These data indicate that G-CSF-induced enhanced mobilization of bone marrow-derived mesenchymal stem cells into BMDCPCs does not change with the nontransdifferentiation of BMDCPCs into adipocytes in several adipose tissues in vivo.

$B M D C P C s$ do not transdifferentiate into adipocytes in regrowing adipose tissues and wound-healing dermal tissue. We next addressed the commitment, if any, of BMDCPCs to transdifferentiate into adipocytes in adipose tissues during regrowth. To create a model of regrowing adipose tissue, we dissected the proximal portion (20\% of the total length) of the left-side epididymal adipose tissue (Figure 6, A and E), and the mice were fed normal or high-fat diet for 2 months following the dissection. Mice fed the high-fat diet exhibited an enhanced regrowth that was approximately $32.7 \%$ more than that of the mice fed the normal diet ( $n=4$ per group). Nevertheless, even in the mice fed the high-fat diet, no $\mathrm{GFP}^{+}$cells were perilipin ${ }^{+}$ adipocytes in any area, including the regrowth and central regions of the left-side epididymal adipose tissue at 2 weeks and 4 months following dissection (Figure 6, B and F-I). In fact, several ( 15 of 

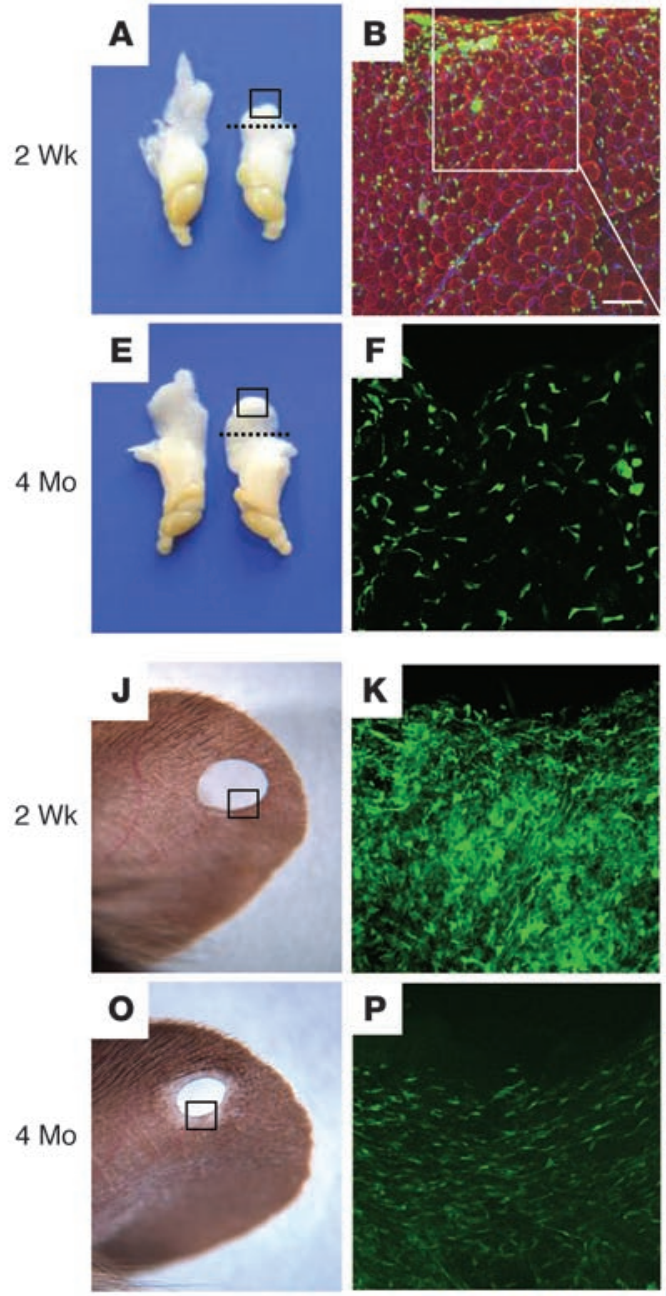
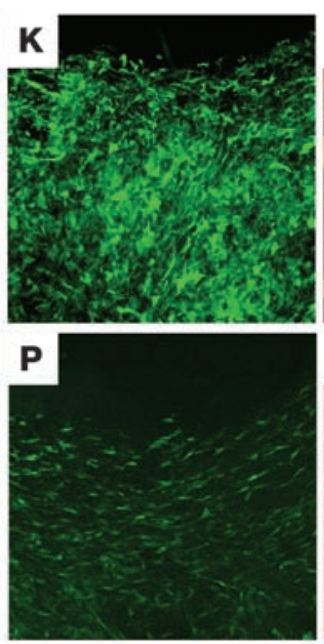
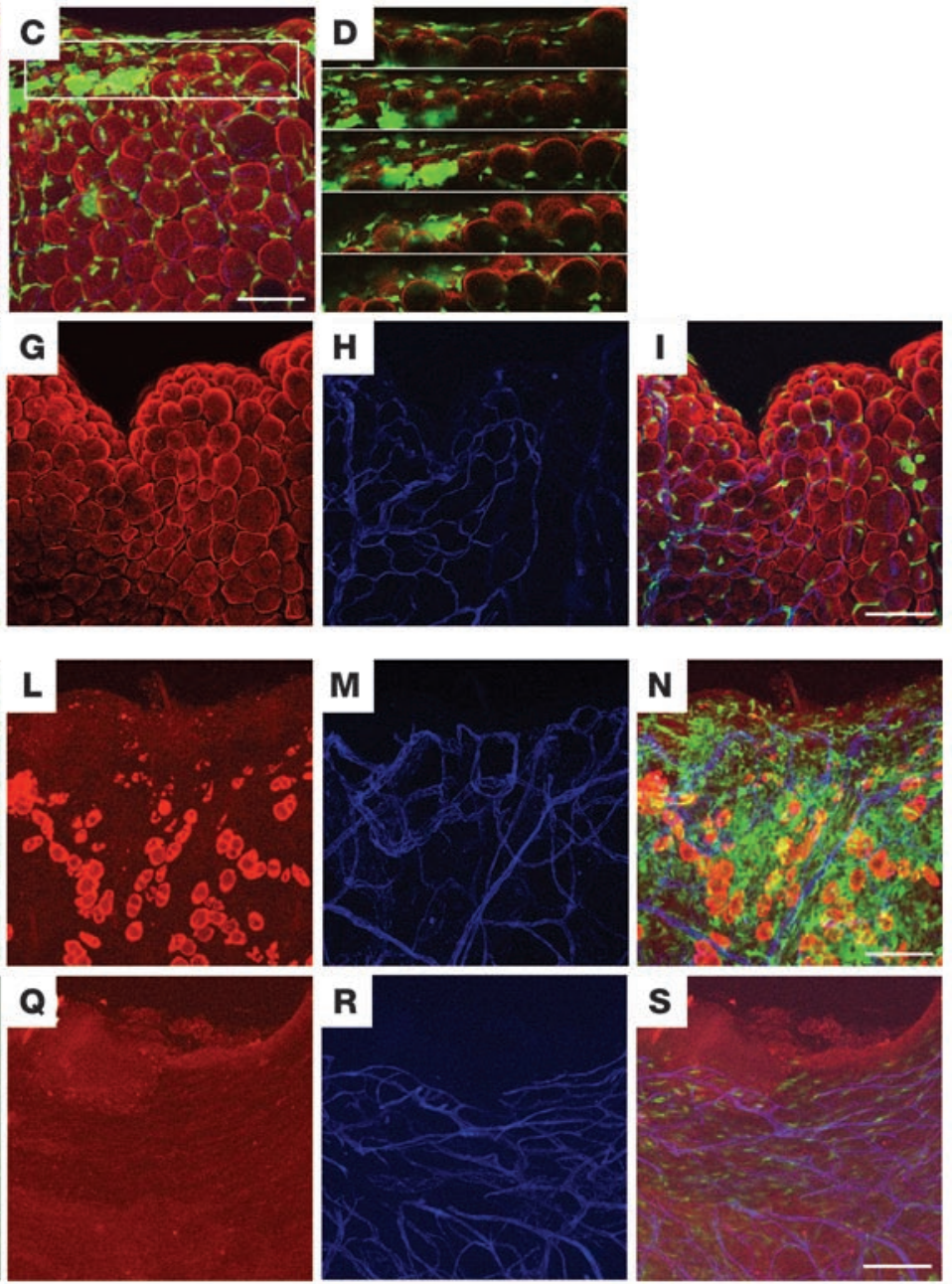

\section{Figure 6}

No GFP+perilipin+ adipocytes were detected in regenerative adipose tissues and wound-healing dermal tissue. (A-I) A proximal portion (20\% of the total length) of the left-side epididymal adipose tissue of 8-week-old C57BL/6J mice that had received BMT from GFP+ mice 2 months before the dissection (dotted lines, $\mathbf{A}$ and $\mathbf{E}$ ). The mice were fed a high-fat diet for the indicated periods. (B-D and F-I) Both sides of the epididymal adipose tissue from the mice were harvested, photographed, and immunostained for perilipin (red stain) and PECAM-1 (blue stain) of the dissected region of the left epididymal adipose tissue (A and E, boxed regions). Some clustered GFP+ cells (green stain) looked like unilocular adipocytes (B and $\mathbf{C}$ ), but the sequential dissected images of this portion (boxed region in $\mathbf{C}$ ) revealed smaller and clustered GFP+perilipin- cells (D). No GFP+perilipin+ adipocytes were detected in the adipose tissues. (J-S) Hole-punch injuries made in the ears of 8-week-old C57BL/6J mice that had received BMT from GFP+ mice 2 months before and were then fed a high-fat diet for the indicated times. Ears from the mice were harvested and photographed, and immunostained for perilipin and PECAM-1 at the healing region of ear ( $\mathbf{J}$ and $\mathbf{O}$, boxed regions). (K-N and P-S) No GFP+ cells were perilipin ${ }^{+}$adipocytes in the wound-healing dermal tissues after the injury. Scale bars: $100 \mu \mathrm{m}$.

40,000 adipocytes) $\mathrm{GFP}^{+}$cells that were similar to unilocular adipocytes were detected in the regrowing region at 2 weeks after the dissection (Figure 6, B and C), but dissecting image analysis of these cells revealed that they were not $\mathrm{GFP}^{+}$unilocular adipocytes, but rather clustered $\mathrm{GFP}^{+}$macrophages and leukocytes.

We also assessed the commitment, if any, of BMDCPCs to transdifferentiate into adipocytes in adult dermal tissues during skin wound healing. To create a dermal wound healing model, we made hole-punch injuries to the ears of adult mice (Figure 6, J and O) and fed them with the normal and high-fat diets as described above. Nevertheless, even in the high-fat diet-fed mice, no $\mathrm{GFP}^{+}$cells were perilipin ${ }^{+}$adipocytes in any area, including the wound-healing and adjacent normal regions of the ear skin at 2 weeks and 2 months after the induced injury ( $n=3$ per group; Figure $6, \mathrm{~K}-\mathrm{N}$ and P-S). In fact, many bone marrow-derived $\mathrm{GFP}^{+}$cells were detected in the healing region at 2 weeks after the injury. However, the number of $\mathrm{GFP}^{+}$cells was markedly reduced and the healing region replaced with scar tissue over time (Figure 6, K and P). These results indicate that BMDCPCs do not transdifferentiate into adipocytes in regrowing adipose tissue and wound-healing dermal tissue.

Bone marrow cells transdifferentiate into multilocular adipocytes in vitro, but not in vivo. The above results raised the question of whether the $\mathrm{GFP}^{+}$cells in the recipient bone marrow could transdifferentiate into adipocytes. To examine this possibility, we isolated the $\mathrm{GFP}^{+}$cells from the recipient bone marrow and cultured them in control or adipogenic medium for 5 days or mixed with 


\section{A}

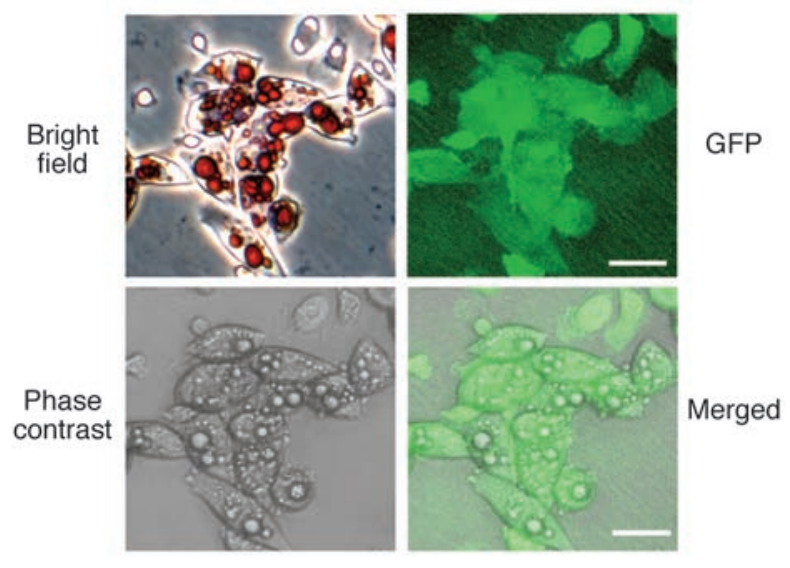

\section{B}
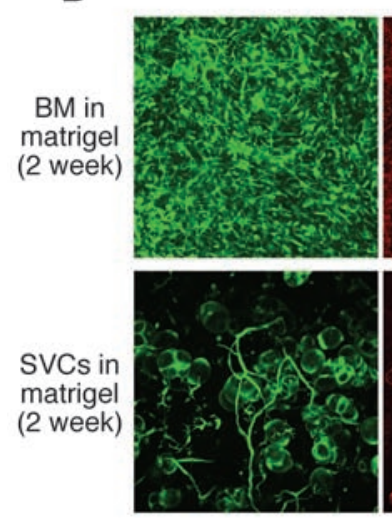

Perilipin

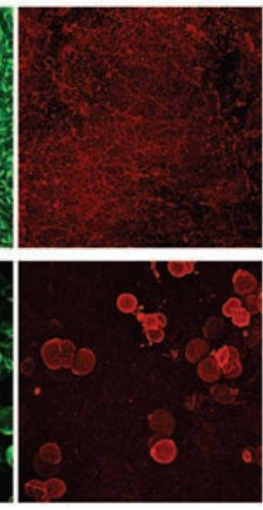

C
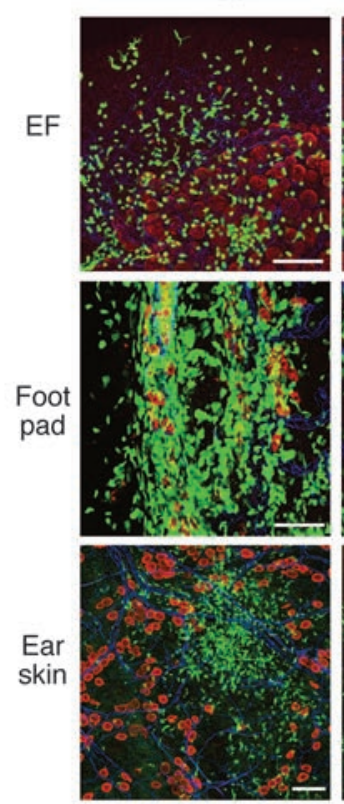

2 week
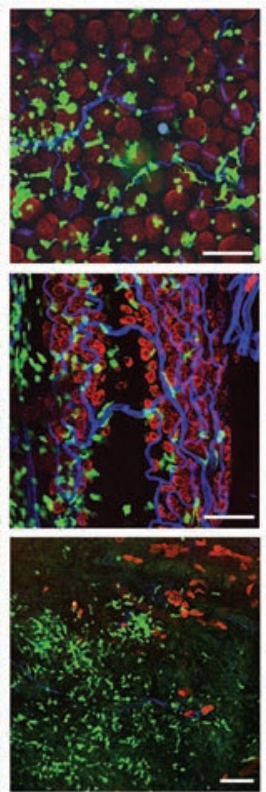

3 week
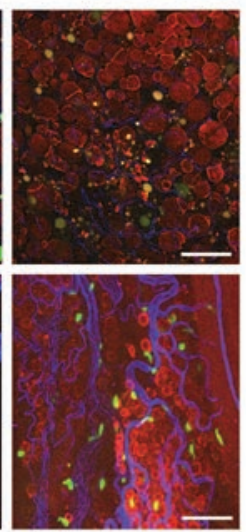

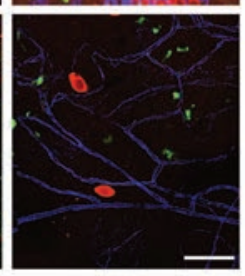

\section{Figure 7}

Bone marrow cells transdifferentiated into multilocular adipocytes in vitro, but not in vivo. Approximately $3 \times 10^{5} \mathrm{GFP}+$ cells from bone marrow of $\mathrm{GFP}^{+}$mice were cultured in the adipogenic medium for 5 days (A), mixed with Matrigel and implanted into the flank region subcutaneously (B), or directly implanted into the epididymal adipose tissue, foot pad, and intradermal region of ear skin (C) of 8-week-old C57BL/6J mice. (B) As a positive control, about $3 \times 10^{5} \mathrm{GFP}+$ SVCs from the epididymal adipose tissue of the GFP + mice were mixed with the Matrigel and implanted into the flank region subcutaneously. (A) The cultured cells were stained with Oil red and hematoxylin, and the images were captured by brightfield, fluorescence, and phase-contrast microscopy and merged. After 2 weeks (B) or the indicated time periods (C), the gels and tissues implanted with the GFP+ cells were harvested, whole-mounted, and immunostained for perilipin (red stain). No GFP+perilipin+ unilocular adipocytes were detected in the gel containing GFP+ (green stain) bone marrow cells, while many GFP+perilipin+ unilocular adipocytes were detected in the gel containing GFP+ SVCs. The shapes of the implanted GFP+ bone marrow cells in the indicated tissues had changed, and the numbers of the cells were markedly reduced over time. No GFP+perilipin ${ }^{+}$adipocytes were detected in the cells. In C, blue stain denotes PECAM-1. Scale bars: $25 \mu \mathrm{m}(\mathbf{A}) ; 100 \mu \mathrm{m}$ (B and C).

Matrigel and implanted into the flank region subcutaneously. The cultured $\mathrm{GFP}^{+}$cells formed small lipid droplet-containing multilocular, but not unilocular, adipocytes in the adipogenic medium (Figure 7A), whereas they did not form lipid droplet-containing cells in the control medium. At 2 weeks after the implantation, no $\mathrm{GFP}^{+}$perilipin ${ }^{+}$adipocytes were found in the Matrigel (Figure 7B). In contrast, stromal vascular cells (SVCs) from epididymal adipose tissue of $\mathrm{GFP}^{+}$mice became unilocular adipocytes in the Matrigel at 2 weeks after the implantation (Figure 7B). These data clearly indicate that bone marrow stem cells rarely become adipocytes in vivo. To test whether bone marrow stem cells per se have no adipogenic potential in vivo, we directly implanted $\mathrm{GFP}^{+}$ bone marrow cells into the epididymal adipose tissue, foot pad, and intradermal region of the ear skin of adult normal mice. The shapes of implanted $\mathrm{GFP}^{+}$bone marrow cells changed, and the numbers of cells were markedly reduced over time (Figure 7C). However, none of the $\mathrm{GFP}^{+}$cells were either unilocular or multilocular adipocytes in epididymal adipose tissue ( $~ 30,000$ adipocytes from 3 mice), food pad ( 3,000 adipocytes from 3 mice), or ear skin ( 3,000 adipocytes from 3 mice; Figure $7 C)$. These results clearly indicate that bone marrow cells do not transdifferentiate into adipocytes under any conditions or in any tissues in vivo.

Evidence that $\mathrm{GFP}^{+}$circulating cells do not transdifferentiate into adipocytes of the adult adipose tissues in the parabiosis model. To confirm the inability of BMDCPCs to transdifferentiate into adipocytes in the BMT model, we assessed the commitment, if any, of BMDCPCs to transdifferentiate into adipocytes in adult adipose tissues using a parabiosis model (35). Consistent with previous reports (36), cross-circulation was established by day 3 after the parabiosis surgical joining, as evidenced by the fact that $\mathrm{GFP}^{+}$cells were detected in several organs, 
A

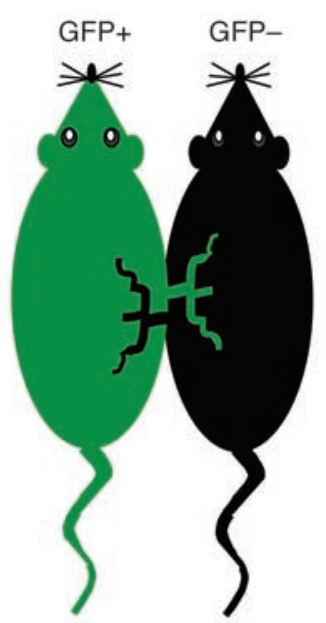

B
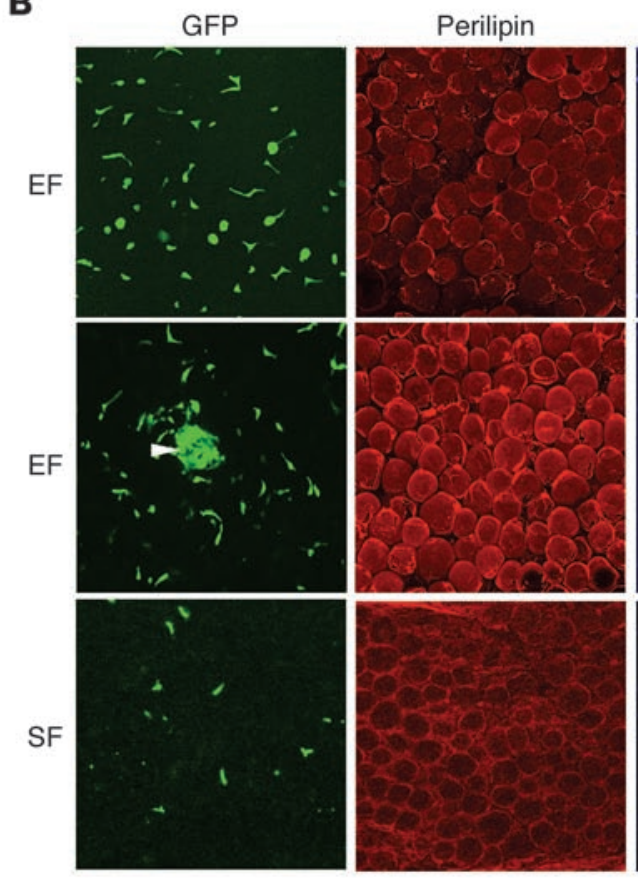

C
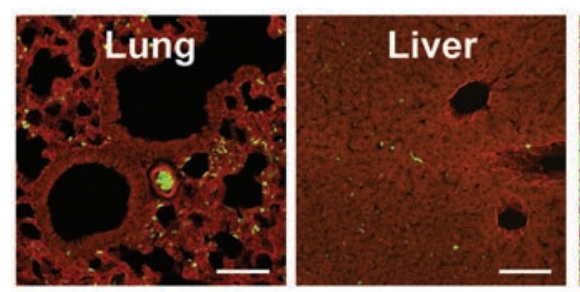
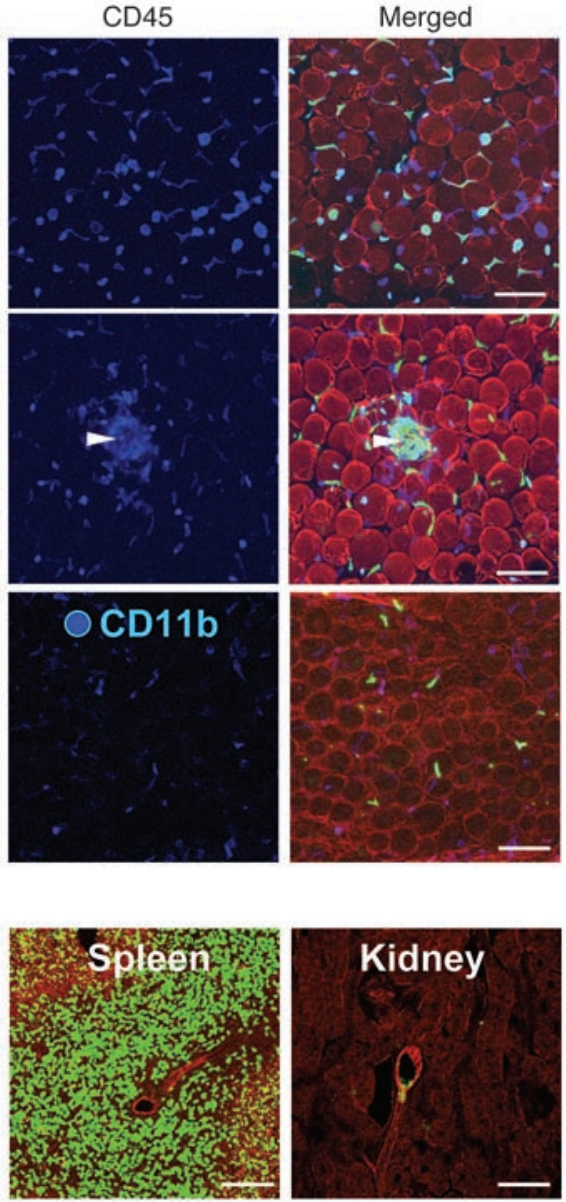

Figure 8

No GFP+perilipin+ adipocytes were detected in the adipose tissues of the parabiosis mouse. (A) The parabiosis between GFP+ and GFP- C57BL/ $^{+}$ $6 \mathrm{~J}$ mice was conducted, with the animals remaining alive for 2 weeks with a normal diet. (B and $\mathbf{C})$ The indicated tissues were harvested, wholemounted, coimmunostained for perilipin (red stain) and CD45 or CD11b (blue stain, B) or perilipin and PECAM-1 (blue stain, C), and merged. No GFP + cells were perilipin ${ }^{+}$adipocytes in the adipose tissues, and clustered GFP ${ }^{+}$CD $45+$ macrophages were detected (white arrowheads). The $\mathrm{GFP}^{+}$cells (green stain) were largely detected in spleen. Scale bars: $100 \mu \mathrm{m}$.

including the adipose tissue and skin dermal tissue (Supplemental Figure 8). We examined $\mathrm{GFP}^{+}$cells in the adipose tissues of $\mathrm{GFP}^{-}$mice transplanted through parabiosis for 2 weeks with $\mathrm{GFP}^{+} \mathrm{BMDCPCs}$ from $\mathrm{GFP}^{+}$mice (Figure 8A). None of the $\mathrm{GFP}^{+}$cells were unilocular or multilocular adipocytes in epididymal ( 30,000 adipocytes from 3 mice; Figure $8 \mathrm{~B})$, mesenteric ( $\sim 18,000$ adipocytes from 3 mice $)$, retroperitoneal ( $\sim 12,000$ adipocytes from 3 mice $)$, subcutaneous $(\sim 30,000$ adipocytes from 3 mice), or dermal ( $\sim 3,000$ adipocytes from 3 mice) adipose tissues. Most $\mathrm{GFP}^{+}$cells were $\mathrm{CD} 11 \mathrm{~b}^{+}$macrophages and $\mathrm{CD}^{2} 5^{+}$leukocytes in the adipose tissues (Figure 8B). Clustered $\mathrm{GFP}^{+}$ cells were often detected (Figure 8B), but these cells were neither multilocular adipocytes nor unilocular adipocytes. In comparison, bone marrow-derived $\mathrm{GFP}^{+}$cells were largely detected in spleen and rarely detected in lung, liver, or kidney as macrophages or lymphocytes (Figure 8C). These results confirm that circulating stem cells do not contribute to form adipocytes in adult adipose tissues.

\section{Discussion}

Our results clearly demonstrated that BMDCPCs did not transdifferentiate into either unilocular or multilocular adipocytes in any normal adipose tissues or in the obese, ROSI-induced multilocularized, and regrowing adipose tissues. We adopted a BMT model transplanted with $\mathrm{GFP}^{+}$bone marrow cells to investigate whether BMDCPCs could transdifferentiate into adipocytes of adult adipose tissues. Visualization of genetically tagged $\mathrm{GFP}^{+}$ cells in whole-mounted adipose tissues using confocal microscopy provided an unambiguous visualization of the cell shapes and localization of BMDCPCs in the multicellular adipose tissues. Moreover, the addition to the whole-mounted adipose tissues of immunostaining for perilipin and collagen IV is critical and of paramount importance for assessing the presence or absence of $\mathrm{GFP}^{+}$perilipin ${ }^{+}$transdifferentiated unilocular and multilocular adipocytes in the adipose tissues. Because perilipin is found exclusively on the outer surface of lipid storage droplets in every adipocyte (26), the visualization of perilipin by specific immunostaining clearly distinguished adipocytes from nonadipocytes in multicellular adipose tissues. Moreover, because collagen IV surrounds each individual adipocyte as a basement membrane (29), the visualization of collagen IV and perilipin by coimmunostaining clearly distinguished multilocular adipocytes from unilocular adipocytes. 
Using these reliable and convincing visualization methods, despite examining more than 900,000 adipocytes in the different adipose tissues, we found no compelling evidence that any of these $\mathrm{GFP}^{+}$ BMDCPCs transdifferentiated into either unilocular or multilocular perilipin ${ }^{+}$adipocytes regardless of their location in the normal, obese, ROSI-treated, or G-CSF-treated mice, even in regrowing or wound-healing adipose tissues. Although adipophilin is known to be expressed ubiquitously in adipose tissue (26-28), our histological analyses revealed that only a certain subpopulation of smallsized adipocytes uniquely and strongly expressed adipophilin in these tissues. These additional findings enabled us to confirm that none of the $\mathrm{GFP}^{+}$BMDCPCs transdifferentiated into adipophilin ${ }^{+}$ adipocytes in the adipose tissues of the normal and obese mice. We also confirmed these results with the parabiosis model.

Our findings are in marked contrast to those of Crossno et al. (25), who recently reported that BMDCPCs could transdifferentiate into multilocular, but not unilocular, adipocytes in the adipose tissues. They adopted a BMT model similar to the one we used: donor bone marrow cells from the transgenic mice that express $\mathrm{GFP}^{+}$on a universal promoter and subjected them to similar protocols of a highfat diet and the ROSI treatment. With this model, the authors found that BMDCPCs transdifferentiated into multilocular adipocytes and that these cells constituted approximately $7 \%-17 \%$ and $15 \%-30 \%$ of total cells in the adipose tissues of the high-fat diet-induced obese and ROSI-treated mice; these cells constituted about $2 \%-7 \%$ of total cells in the adipose tissues of the normal mice. However, their conclusions were based entirely on data derived from flow cytometric analysis of adipocyte suspensions and sectioned adipose tissues for assessment of the $\mathrm{GFP}^{+}$cells, without coimmunostaining of perilipin, collagen IV, or macrophage markers. Thus, they may not have clarified the identity of the "bunch" shape of clustered $\mathrm{GFP}^{+}$cells (25) and "adipocyte-like" clustered $\mathrm{GFP}^{+}$macrophages adequately and accurately in the adipose tissues.

Throughout our assessments, we observed approximately 1,000 clustered adipocyte-like $\mathrm{GFP}^{+}$cells in the adipose tissues of obese, ROSI-treated, and G-CSF-treated mice and in regrowing adipose tissue. Dissecting image analysis of these cells by confocal microscopy, however, revealed that these cells were clustered $\mathrm{GFP}^{+}$macrophages rather than $\mathrm{GFP}^{+}$adipocytes. Interestingly, these clustered $\mathrm{GFP}^{+}$macrophages strongly expressed F4/80, expressed CD45 and CD11b to a lesser extent, and expressed LYVE-1 at low levels. However, Crossno et al. (25) likely interpreted these clustered adipocytelike $\mathrm{GFP}^{+}$macrophages as $\mathrm{GFP}^{+}$multilocular adipocytes. The clustered macrophages in obese adipose tissues are known to be active phagocytic macrophages on dead adipocytes (37). In this regard, $\mathrm{F} 4 / 80^{+}$macrophages could have more enhanced phagocytic activity than do LYVE- $1^{+}$macrophages. It is known that increased infiltration of $\mathrm{F} 4 / 80^{+}$and $\mathrm{CD} 11 \mathrm{~b}^{+}$macrophages in the adipose tissue contributes to the development of metabolic syndrome (38-40). We recently found that LYVE- $1^{+}$macrophages in the tip region of epididymal adipose tissue have an angiogenic role (41). Thus, macrophages expressing different markers may have different functions in the adipose tissue. It is intriguing to note that pharmacological activation of PPAR $\gamma$ with ROSI induced marked and characteristic multilocularization of adipocytes in most white adipose tissues. Adipocyte multilocularization can be found in special situations such as $\beta 3$-adrenergic stimulation $(42,43)$, exposure to cold temperature (44), or deletion of translational inhibitor 4E-BP1 (45). Although the underlying mechanism that creates the adipocyte multilocularization by the ROSI treatment and the significance of adipocyte multilocularization in regulation of lipid storage remain unclear, the multilocularization process occurs from unilocular adipocytes within adipose tissue, but not outside of it.

Consistent with a wealth of previous findings $(30,46,47)$, our study shows that in vitro cultured $\mathrm{GFP}^{+}$progenitor cells from bone marrow can transdifferentiate into multilocular, but not into unilocular, adipocytes in adipogenic medium. In contrast, however, we found no compelling evidence that bone marrow stem cells per se transdifferentiate into adipocytes in the implanted Matrigel or in tissues into which they were directly implanted. Moreover, even though the BMDCPCs contained more mobilized bone marrow-derived mesenchymal stem cells due to the G-CSF treatment, there was no transdifferentiation of BMDCPCs into adipocytes in the adipose tissues in vivo. In comparison, the SVCs derived from $\mathrm{GFP}^{+}$mice differentiated into unilocular adipocytes in the implanted Matrigel. Thus, BMDCPCs could have adipogenic potential in vitro in a highly calorie-preserving adipogenic medium, but not in any situations in vivo. To date, controversial findings from one extreme to the other are still appearing as to whether BMDCPCs can give rise to cardiomyocytes $(20,21,48)$, smooth muscle (22), skeletal muscle (15), vascular endothelial cells $(17,18)$, renal tubular epithelial cells $(49)$, kidney mesangial cells (19), hepatocytes (16), or cardiac valve interstitial cells (23) in pathologic and regenerative tissues in vivo. Thus, BMDCPCs may or may not give rise many different resident cells in every organ, ranging from differentiated to undifferentiated cells, depending on the microenvironment. One rare case of reverse adipocyte-toepithelium transdifferentiation takes place in the mammary gland of adult mice during pregnancy and lactation (50).

Regardless of findings in other tissues, our results indicate that BMDCPCs do not give rise any differentiated resident adipocytes, even in regenerative adipose tissues and wound-healing dermal tissue. These results have led to some intriguing questions. It is still unknown whether there are any determined adipocyte stem cells or progenitors in bone marrow, which can be properly mobilized by certain stimuli into systemic circulation to become adipoblasts or preadipocytes via transdifferentiation triggered by further appropriate stimuli. If these cells do exist in bone marrow, do adipose tissues provide an unfavorable environment for their homing or transdifferentiation? To address this hypothesis, we need to identify selective markers that can distinguish adipoblasts or preadipocytes in bone marrow, circulating blood, and adipose tissues.

Finally, the parabiosis model used in this study confirmed that BMDCPCs cannot transdifferentiate into adipocytes. This model circumvented the disadvantages of the BMT model regarding the age of the mice, possible loss of mesenchymal stem cell repopulation in bone marrow, and unavoidable radiation injury on bone marrow. The age of the mice receiving BMT was relatively high at 6-8 months of age when the adipose tissues were analyzed after several treatments; therefore, the transdifferentiation capability of BMDCPCs could have been markedly reduced. However, the age of the mice receiving parabiosis was only $2-3$ months when the adipose tissues were analyzed. In the BMT model, it is possible that a number of mesenchymal stem cells responsible for adipocyte stem cells could be reduced in bone marrow during repopulation by procedures of whole-body irradiation and systemic BMT. Moreover, these procedures result in improper mobilization of adipogenic bone marrow stem cells from bone marrow. In comparison, in the parabiosis model, intact donor BMDCPCs derived from the intact bone marrow are directly circulated into syngeneic recipient 
circulation, homing to several organs including adipose tissues. Nevertheless, using this parabiosis model, we found no compelling evidence that BMDCPCs can transdifferentiate into adipocytes.

Taken together, our results provide evidence that BMDCPCs do not transdifferentiate into either unilocular or multilocular adipocytes in the adipose tissues. Therefore, based on these results, we conclude that BMDCPCs play a negligible role in expanding the number of adipocytes in the growth of adipose tissues.

\section{Methods}

Mice. Specific pathogen-free C57BL/6J (C57BL/6J genetic background) mice were purchased from The Jackson Laboratory. $\mathrm{GFP}^{+}$mice (C57BL/6J genetic background) were a gift from M. Okabe (Osaka University, Osaka, Japan). Mice were bred in our pathogen-free animal facility, and male mice aged about 8-9 weeks were used for this study unless otherwise indicated. Animal care and experimental procedures were performed under approval from the Animal Care Committees of KAIST and Dankook University. All animals were fed a standard normal diet (PMI LabDiet; Purina Mills Inc.) ad libitum with free access to water.

Isolation of bone marrow cells and BMT. Bone marrow cells $\left(2 \times 10^{6}\right)$ were harvested from the femurs and tibias of $\mathrm{GFP}^{+}$mice by flushing with ice-cold Dulbecco's PBS (DPBS; Sigma-Aldrich). The recipient mice (C57BL/6J), 8 weeks old, were sublethally irradiated at a dose of 4.5 Gy with a gamma irradiator (Gammacell 3000; MDS Nordion Inc.). Bone marrow cells were then injected intravenously into the recipient mice 16 hours after irradiation. The indicated tissues of BMT mice were examined at the indicated times (41).

Treatments. All treatments were performed at approximately 2 months after BMT. For studies of obesity induced by a high-fat diet, mice were fed with a high-fat diet (34.9\%, wt/wt, catalog no. D12492; Research Diets Inc.) for the 2-month period. For ROSI treatment, the mice were fed normal diet food containing ROSI (SmithKline Beecham Pharmaceuticals) ad libitum $(15 \mathrm{mg} / \mathrm{kg} / \mathrm{d})$ for 4 weeks. To further mobilize mesenchymal stem cells from bone marrow to BMDCPCs, the mice were treated with G-CSF (intraperitoneal injection, $10 \mu \mathrm{g} / \mathrm{kg} / \mathrm{d}$; Choongwae Pharma Corporation) for 2 weeks. To develop a regenerative adipose tissue model, we anesthetized the mice by intramuscular injection of a combination of anesthetics (80 mg/kg ketamine and $12 \mathrm{mg} / \mathrm{kg}$ xylazine) and dissected without macroscopic bleeding the proximal portion ( $20 \%$ of the total length) of the leftside epididymal adipose tissue. To produce a regenerative dermal tissue model, we made a $2.0-\mathrm{mm}$ hole in the center of both ears of each mouse using a metal ear punch (Harvard Apparatus) (51).

Mesenchymal stem cell isolation and culture. Bone marrow was collected from 8- to 10 -week-old C57BL/6 and $\mathrm{GFP}^{+}$mice by flushing femurs and tibias with ice-cold DPBS (Sigma-Aldrich). The cells were then washed twice in complete medium (52) consisting of DMEM-low glucose (Cambrex), $2 \mathrm{mM}$ L-glutamine, $10 \%$ fetal bovine serum (Hyclone), and penicillin/streptomycin $(5 \mathrm{U} / \mathrm{ml}$; Invitrogen) and plated on a Petri dish at a density of $2 \times 10^{6}$ cells $/ \mathrm{cm}^{2}$. After 5 days, nonadherent cells were removed by $2-3$ washes with DPBS and adherent cells further cultured in the complete medium. The cultured mesenchymal stem cells were differentiated into adipocytes using the Mesenchymal Stem Cell Adipogenesis Kit (Chemicon International Inc.) according to the manufacturer's instructions.

Implantation of bone marrow cells and SVCs. Bone marrow cells and SVCs from epididymal adipose tissue were harvested from $\mathrm{GFP}^{+}$mice as previously described (41). Briefly, to obtain SVCs, the tip portion of epididymal adipose tissue (41) was harvested and incubated with Hanks' balanced salts solution (Sigma-Aldrich) containing 0.2\% type 2 collagenase (Worthington) for 1 hour at $37^{\circ} \mathrm{C}$. After inactivation of collagenase activity with bovine serum, the cell suspension was filtered through a $100-\mu \mathrm{m}$ nylon filter (BD Biosciences) and centrifuged at $400 \mathrm{~g}$ for $5 \mathrm{~min}$. The floating adipo- cytes were separated from pelleted SVCs, and both cell types were washed and resuspended with DPBS. Lack of perilipin ${ }^{+}$-differentiated adipocytes in the suspended SVCs was confirmed by immunofluorescence staining. A total of $3 \times 10^{5}$ bone marrow cells or SVCs were mixed with Matrigel (100 $\mu \mathrm{l}$; BD Biosciences) and implanted into the flank region subcutaneously and directly implanted into the epididymal adipose tissue, foot pad, and intradermal region of the ear skin of 8-week-old C57BL/6J mice.

Parabiosis model. Pairs of 6- to 10-week-old wild-type and $\mathrm{GFP}^{+}$mice were subjected to parabiotic surgery. Mice were anesthetized by intramuscular injection of the combination of anesthetics $(80 \mathrm{mg} / \mathrm{kg}$ ketamine and 12 $\mathrm{mg} / \mathrm{kg}$ xylazine), then surgically joined from shoulder to femur. At the indicated number of weeks after parabiotic surgery, $\mathrm{GFP}^{+}$cells in the adipose tissues of wild-type mice were examined.

Histological and morphometric analysis. Mice were anesthetized by intramuscular injection of the combination of anesthetics, and adipose tissues were fixed by vascular perfusion of $1 \%$ paraformaldehyde in PBS and whole-mounted or cryo-embedded and sectioned. Whole-mount tissues and cryosections ( $20 \mu \mathrm{m}$ thickness) were prepared and incubated for $1 \mathrm{~h}$ at room temperature with blocking solution containing $5 \%$ goat serum (Jackson ImmunoResearch Laboratories Inc.) in PBST (0.3\% Triton X-100 in PBS). After blocking, the whole-mounted tissues and cryosections were incubated overnight at $4^{\circ} \mathrm{C}$ with one or more of the following primary antibodies: (a) for adipocytes, guinea pig anti-perilipin antibody (diluted 1:1,000; Research Diagnostics) and guinea pig anti-adipophilin antibody (diluted 1:1,000; Research Diagnostics); (b) for blood vessels, hamster anti-PECAM-1 antibody (clone 2H8, diluted 1:1,000; Chemicon International); (c) for macrophages and leukocytes, rat anti-mouse CD11b antibody (clone M1/70, diluted 1:1,000; BD Biosciences - Pharmingen), rat anti-mouse F4/80 antibody (clone Cl:A3-1, diluted 1:1,000; Serotec), rat anti-mouse LYVE-1 antibody (clone Han-1, diluted 1:1,000; Aprogen), and rat anti-mouse CD45 (clone 30-F11, diluted 1:1,000; BD Biosciences - Pharmingen); and (d) for basement membrane of individual adipocytes, rabbit anti-collagen IV antibody (diluted 1:1,000; Cosmo Bio Co. Ltd.). After several washes in PBST, whole-mounted tissues and cryosections were incubated for $1 \mathrm{~h}$ at room temperature with one or more secondary antibodies: (a) Cy3- or Cy5-conjugated anti-guinea pig antibody (Jackson ImmunoResearch Laboratories); (b) Cy3- or Cy5-conjugated anti-rat antibody or anti-rabbit antibody (diluted 1:500; Jackson ImmunoResearch Laboratories); or (c) Cy3- or Cy5-conjugated anti-hamster IgG antibody (diluted 1:500; Jackson ImmunoResearch Laboratories). For control experiments, the primary antibody was omitted or substituted with preimmune serum. Signals were visualized, and digital images were obtained using a Zeiss ApoTome microscope and a Zeiss LSM 510 confocal microscope equipped with argon and helium-neon lasers (Carl Zeiss). For determining the $\mathrm{GFP}^{+}$unilocular and multilocular adipocytes, double-immunostained color images for perilipin and collagen IV were captured with a Zeiss LSM 510 confocal microscope. Using ImageJ software (http://rsb.info.nih. gov/ij), we selected the clustered $\mathrm{GFP}^{+}$area as a region of interest from macrophage/ $\mathrm{GFP}^{+}$-stained images, and macrophage/GFP ${ }^{+}$-stained images were converted to 8-bit gray scale. Area densities of the macrophage/GFP+stained images were measured from the pixels in the region of interest; only pixels exceeding a certain level (>50 intensity value) were taken to exclude background fluorescence.

Statistics. Values presented are means \pm SD. Significant differences between means were determined by analysis of variance followed by the StudentNewman-Keuls test. Statistical significance was set at $P<0.05$ or $P<0.01$.

\section{Acknowledgments}

This work was supported by the Korea Science and Engineering Foundation (KOSEF) through the National Research Laboratory 
Program (2004-02376 to G.Y. Koh) funded by the Ministry of Science and Technology.

Received for publication April 25, 2007, and accepted in revised form September 19, 2007.

1. Greenberg, A.S., and Obin, M.S. 2006. Obesity and the role of adipose tissue in inflammation and metabolism. Am. J. Clin. Nutr. 83:461S-465S.

2. Shoelson, S.E., Lee, J., and Goldfine, A.B. 2006. Inflammation and insulin resistance. J. Clin. Invest. 116:1793-1801.

3. Martin, R.J., Hausman, G.J., and Hausman, D.B. 1998. Regulation of adipose cell development in utero. Proc. Soc. Exp. Biol. Med. 219:200-210.

4. Hausman, D.B., DiGirolamo, M., Bartness, T.J., Hausman, G.J., and Martin, R.J. 2001. The biology of white adipocyte proliferation. Obes. Rev. 2:239-254.

5. Rosen, E.D., and MacDougald, O.A. 2006. Adipocyte differentiation from the inside out. Nat. Rev. Mol. Cell Biol. 7:885-896.

6. Schutz, Y. 2004. Dietary fat, lipogenesis and energy balance. Physiol. Behav. 83:557-564.

7. Crandall, D.L., Hausman, G.J., and Kral, J.G. 1997. A review of the microcirculation of adipose tissue: anatomic, metabolic, and angiogenic perspectives. Microcirculation. 4:211-232.

8. Hausman, G.J., and Richardson, R.L. 1983. Cellular and vascular development in immature rat adipose tissue. J. Lipid Res. 24:522-532.

9. Zuk, P.A., et al. 2002. Human adipose tissue is a source of multipotent stem cells. Mol. Biol. Cell. 13:4279-4295.

10. Cousin, B., Andre, M., Arnaud, E., Penicaud, L., and Casteilla, L. 2003. Reconstitution of lethally irradiated mice by cells isolated from adipose tissue. Biochem. Biophys. Res. Commun. 301:1016-1022.

11. Prunet-Marcassus, B., et al. 2006. From heterogeneity to plasticity in adipose tissues: site-specific differences. Exp. Cell Res. 312:727-736.

12. Cinti, S. 2001. The adipose organ: morphological perspectives of adipose tissues. Proc. Nutr. Soc. 60:319-328.

13. Cinti, S. 2005. The adipose organ. Prostaglandins Leukot. Essent. Fatty Acids. 73:9-15.

14. Faust, I.M., Johnson, P.R., Stern, J.S., and Hirsch, J. 1978. Diet-induced adipocyte number increase in adult rats: a new model of obesity. Am. J. Physiol. 235:E279-E286.

15. Camargo, F.D., Green, R., Capetanaki, Y., Jackson, K.A., and Goodell, M.A. 2003. Single hematopoietic stem cells generate skeletal muscle through myeloid intermediates. Nat. Med. 9:1520-1527.

16. Dalakas, E., Newsome, P.N., Harrison, D.J., and Plevris, J.N. 2005. Hematopoietic stem cell trafficking in liver injury. FASEBJ. 19:1225-1231.

17. Jackson, K.A., et al. 2001. Regeneration of ischemic cardiac muscle and vascular endothelium by adult stem cells. J. Clin. Invest. 107:1395-1402.

18. Kinnaird, T., Stabile, E., Burnett, M.S., and Epstein, S.E. 2004. Bone-marrow-derived cells for enhancing collateral development: mechanisms, animal data, and initial clinical experiences. Circ. Res. 95:354-363.

19. Masuya, M., et al. 2003. Hematopoietic origin of
Address correspondence to: Gou Young Koh, Department of Biological Sciences, KAIST, 373-1, Guseong-dong, Daejeon, 305-701, Republic of Korea. Phone: 82-42-869-2638; Fax: 82-42-869-2610; E-mail: gykoh@kaist.ac.kr. glomerular mesangial cells. Blood. 101:2215-2218.

20. Orlic, D., et al. 2001. Mobilized bone marrow cells repair the infarcted heart, improving function and survival. Proc. Natl. Acad. Sci. U. S. A. 98:10344-10349.

21. Pittenger, M.F., and Martin, B.J. 2004. Mesenchymal stem cells and their potential as cardiac therapeutics. Circ. Res. 95:9-20.

22. Sata, M., et al. 2002. Hematopoietic stem cells differentiate into vascular cells that participate in the pathogenesis of atherosclerosis. Nat. Med. 8:403-409.

23. Visconti, R.P., et al. 2006. An in vivo analysis of hematopoietic stem cell potential: hematopoietic origin of cardiac valve interstitial cells. Circ. Res. 98:690-696.

24. Okabe, M., Ikawa, M., Kominami, K., Nakanishi, T., and Nishimune, Y. 1997. 'Green mice' as a source of ubiquitous green cells. FEBS Lett. 407:313-319.

25. Crossno, J.T., Jr. Majka, S.M., Grazia, T., Gill, R.G., and Klemm, D.J. 2006. Rosiglitazone promotes development of a novel adipocyte population from bone marrow-derived circulating progenitor cells. J. Clin. Invest. 116:3220-3228.

26. Londos, C., Sztalryd, C., Tansey, J.T., and Kimmel, A.R. 2005. Role of PAT proteins in lipid metabolism. Biochimie. 87:45-49.

27. Robenek, H., Robenek, M.J., and Troyer, D. 2005. PAT family proteins pervade lipid droplet cores. J. Lipid Res. 46:1331-1338.

28. Wolins, N.E., et al. 2005. S3-12, Adipophilin, and TIP47 package lipid in adipocytes. J. Biol. Chem. 280:19146-19155.

29. Martinez-Hernandez, A., and Amenta, P.S. 1983. The basement membrane in pathology. Lab. Invest. 48:656-677.

30. Ryden, M., et al. 2003. Functional characterization of human mesenchymal stem cell-derived adipocytes. Biochem. Biophys. Res. Commun. 311:391-397.

31. Bosnakovski, D., et al. 2005. Isolation and multilineage differentiation of bovine bone marrow mesenchymal stem cells. Cell Tissue Res. 319:243-253.

32. Zeng, L., et al. 2006. Multipotent adult progenitor cells from swine bone marrow. Stem Cells. 24:2355-2366.

33. Kawada, H., et al. 2004. Nonhematopoietic mesenchymal stem cells can be mobilized and differentiate into cardiomyocytes after myocardial infarction. Blood. 104:3581-3587.

34. Fukuda, K., and Fujita, J. 2005. Mesenchymal, but not hematopoietic, stem cells can be mobilized and differentiate into cardiomyocytes after myocardial infarction in mice. Kidney Int. 68:1940-1943.

35. Wagers, A.J., Sherwood, R.I., Christensen, J.L., and Weissman, I.L. 2002. Little evidence for developmental plasticity of adult hematopoietic stem cells. Science. 297:2256-2259.

36. Wright, D.E., Wagers, A.J., Gulati, A.P., Johnson, F.L., and Weissman, I.L. 2001. Physiological migra- tion of hematopoietic stem and progenitor cells. Science. 294:1933-1936.

37. Cinti, S., et al. 2005. Adipocyte death defines macrophage localization and function in adipose tissue of obese mice and humans. J. Lipid Res. 46:2347-2355.

38. Weisberg, S.P., McCann, D., Desai, M., Rosenbaum, M., Leibel, R.L., and Ferrante, A.W., Jr. 2003. Obesity is associated with macrophage accumulation in adipose tissue. J. Clin. Invest. 112:1796-1808.

39. Wellen, K.E., and Hotamisligil, G.S. 2003. Obesityinduced inflammatory changes in adipose tissue. J. Clin. Invest. 112:1785-1788.

40. Xu, H., et al. 2003. Chronic inflammation in fat plays a crucial role in the development of obesity-related insulin resistance. J. Clin. Invest. 112:1821-1830.

41. Cho, C.H., et al. 2007. Angiogenic role of LYVE-1positive macrophages in adipose tissue. Circ. Res. 100:e47-e57.

42. Granneman, J.G., Li, P., Zhu, Z., and Lu, Y. 2005. Metabolic and cellular plasticity in white adipose tissue I: effects of beta3-adrenergic receptor activation. Am. J. Physiol. Endocrinol. Metab. 289:E608-E616.

43. Himms-Hagen, J., et al. 2000. Multilocular fat cells in WAT of CL-316243-treated rats derive directly from white adipocytes. Am. J. Physiol. Cell Physiol. 279:C670-C681.

44. Loncar, D., Afzelius, B.A., and Cannon, B. 1988. Epididymal white adipose tissue after cold stress in rats. II. Mitochondrial changes. J. Ultrastruct. Mol. Struct. Res. 101:199-209.

45. Tsukiyama-Kohara, K., et al. 2001. Adipose tissue reduction in mice lacking the translational inhibitor 4E-BP1. Nat. Med. 7:1128-1132.

46. Gimble, J.M., Zvonic, S., Floyd, Z.E., Kassem, M., and Nuttall, M.E. 2006. Playing with bone and fat. J. Cell. Biochem. 98:251-266.

47. Hong, K.M., Burdick, M.D., Phillips, R.J., Heber, D., and Strieter, R.M. 2005. Characterization of human fibrocytes as circulating adipocyte progenitors and the formation of human adipose tissue in SCID mice. FASEB J. 19:2029-2031.

48. Murry, C.E., et al. 2004. Haematopoietic stem cells do not transdifferentiate into cardiac myocytes in myocardial infarcts. Nature. 428:664-668.

49. Krause, D., and Cantley, L.G. 2005. Bone marrow plasticity revisited: protection or differentiation in the kidney tubule? J. Clin. Invest. 115:1705-1708.

50. Morroni, M., et al. 2004. Reversible transdifferentiation of secretory epithelial cells into adipocytes in the mammary gland. Proc. Natl. Acad. Sci. U. S. A. 101:16801-16806.

51. Cho, C.H., et al. 2006. COMP-angiopoietin-1 promotes wound healing through enhanced angiogenesis, lymphangiogenesis, and blood flow in a diabetic mouse model. Proc. Natl. Acad. Sci. U. S. A. 103:4946-4951.

52. Tropel, P., et al. 2004. Isolation and characterisation of mesenchymal stem cells from adult mouse bone marrow. Exp. Cell Res. 295:395-406. 